\title{
Physiotherapy and Pelvic Floor Muscle Exercises for the Prevention and Treatment of Pregnancy-Related Pelvic Floor Dysfunctions: A Systematic Review and Meta-analysis
}

\author{
Fahime Khorasani ${ }^{1}{ }^{\circledR}$, Fariba Ghaderi ${ }^{12^{*}}{ }^{\circledR}$, Parvin Sarbakhsh $^{3}$, Parisa Ahadi ${ }^{2}$, Elahe Khorasani $^{4}$, Fereshteh \\ Ansari $^{2}$, Nafiseh Vahed $^{2}$
}

\begin{abstract}
Objectives: The present systematic review focused on the prevention or treatment of three main types of pelvic floor dysfunctions (PFDs) specifically pelvic organ prolapse (POP), urinary incontinence (UI), and fecal incontinence (FI) using physiotherapy and pelvic floor muscle exercises (PFMEs). With regard to the breadth of the problem, there is not much evidence grounded on the best management. The main purpose of this systematic review was to evaluate the effects of physiotherapy and PFMEs on the prevention and treatment of pregnancy-related PFDs; namely, POP, FI, and UI. Therefore, this review incorporated studies comparing the use of physiotherapy and PFMEs with every other existing interventions.

Methods: This systematic review and meta-analysis was conducted on randomized-controlled-trial (RCT) articles and quasi-RCT designs through a search in the studies published with no time limits until December 2017 in the databases of PubMed (Medline), Web of Science, Scopus, Embase, Cochrane Library, and ProQuest. The meta-analysis was also applied for data synthesis. Moreover, heterogeneity was assessed using Cochran's Q test and $\mathrm{I}^{2}$ index.

Results: A total number of 26 RCTs were examined in this review in which the outcome variables were related to POP, UI, and FI prevalence; POP, UI, and FI severity, as well as pelvic floor muscle (PFM) strength and endurance. In most articles, UI prevalence or severity in intervention groups had significantly improved compared with those in controls. The number of studies examining POP and FI was also relatively low. In two studies, FI severity or prevalence in intervention groups had significantly enhanced in comparison with those in control groups; however, FI prevalence in two articles had been reported lower in intervention groups than that in control groups although no significant difference had been observed. There was also no significant improvement in intervention groups in two other studies in this respect. Besides, three articles had not reported traces of improvement in POP, as well as a significant difference between intervention and control groups. Nevertheless, two studies had found a significant improvement in POP in this regard. Based on meta-analysis results for the variable of PFM strength, Cochran's Q test $(P<0.001)$ and I2 index (90.02) indicated heterogeneity between studies; so, a random-effect meta-analysis was applied to estimate overall effect sizes. The overall mean differences following intervention between the study groups were also equal to 6.94, with a 95\% CI (1.36 to 12.52 ).

Conclusions: It was concluded that physiotherapy and PFMEs might have effects on pregnancy-related UI, but they had not consistently reduced FI severity or prevalence and failed to constantly improve POP.

Keywords: Physiotherapy, Pelvic floor muscle exercise, Pelvic organ prolapse, Urinary incontinence, Fecal incontinence
\end{abstract}

\section{Introduction}

Pelvic floor dysfunctions (PFDs), viz pelvic organ prolapse (POP), urinary incontinence (UI), and fecal incontinence (FI) characterize the main side effects induced by pregnancy and childbirth $(1,2)$.

Approximately $30 \%-50 \%$ of postpartum women also suffer from UI and 3\%-10\% of them can be affected by FI $(3,4)$. Although there are few studies on postpartum POP, some of them have reported that more than $50 \%$ of women are experiencing mild POP following childbirth $(5,6)$. Given the high prevalence of postpartum PFDs and how they shape quality of life, many researchers have also investigated the effects of different interventions on the prevention and treatment of pregnancy-related PFDs. Thus, a systematic Cochrane review was fulfilled to shed light on the effects of PFMEs on UI prevention and treatment among prenatal and postpartum women and its results revealed that PFMEs were better than no interventions for patients with postnatal incontinence, but intensive and supervised exercises had brought about better effects compared with home-based ones (7); therefore, it is essential to have a specific protocol for each patient (8).

Physiotherapeutic interventions are also recommended 
for the treatment of PFDs. Moreover; PFMEs, biofeedback, stabilization exercises, electrical stimulation, and use of vaginal cones are accessible tools for PFM physiotherapists (5,9-13).

\section{Aim}

The main purpose of this study was to check articles examining the effects of physiotherapy and PFMEs compared with no interventions in terms of prevention and treatment of pregnancy-related PFDs including POP, FI, and UI.

\section{Methods}

The protocol of this systematic review was registered in the international database of prospectively registered systematic reviews in health and social care (PROSPERO; Registred No. CRD42018103654) .

\section{Eligibility Criteria}

This systematic review included all published articles meeting the following criteria:
a. RCTs or studies with a quasi-RCT design
b. Pregnant women with or without POP, UI, and FI
c. Postpartum women with or without POP, UI, and FI
d. Postpartum women undergoing natural childbirth or Cesarean section
e. Different types of physiotherapy intervention methods
f. Control group with or without any treatments
g. Outcome variables related to POP, UI, FI prevalence; POP, UI, FI severity, as well as FFM strength and endurance
h. Published in English; and
i. Published until December 2017
Non-English articles and non-RCTs or those without a quasi-RCT design were excluded.

\section{Search Strategy}

With no time limits until December 2017, the articles published in the databases of PubMed (Medline), Web of Science, Scopus, Embase, Cochrane Library, and Proquest were searched to meet the main purpose of this systematic review.

The search strategy included a combination of Medical Subject Headings (MeSH) and free keywords such as prenatal, postnatal, postpartum, pelvic floor muscle exercise, pelvic floor dysfunction, pelvic organ prolapse, urinary incontinence, and fecal incontinence. The search strategy is accessible in Supplementary file 1.

\section{Study Selection}

In the first step, the titles and abstracts of the published articles were reviewed to exclude the irrelevant ones. Then, in the second step, the abstracts of the included studies were reviewed. Finally, in the third step, the full-texts of the relevant articles were analyzed. Two independent investigators also explored the searched articles to select the ambiguous cases based on the inclusion criteria. The most relevant or recent duplicate studies were enrolled as well.

\section{Data Extraction}

The required data were extracted from the articles included in the review using a modified standardized data extraction tool by two independent reviewers. The extraction table presents information about interventions, populations, study methods, and outcomes of the articles and their significance to the review question and its specific objectives (Supplementary file 2).

Assessment of Methodological Quality

The selected studies were critically appraised by two independent reviewers for their methodological quality using standardized critical appraisal instruments for physiotherapy research from Physiotherapy Evidence Database (PEDro) (14) (Supplementary file 3). Any possible disagreements between reviewers were also resolved via discussions, or recruitment of a third reviewer.

Data Synthesis

The comprehensive meta-analysis (CMA) was used to pool, if possible, the findings of the articles in the statistical meta-analysis. The effect size was also reported as mean differences for continuous data with a 95\% confidence interval (CI). Furthermore, Cochran's $Q$ test and $\mathrm{I}^{2}$ index were used to explore heterogeneity. According to the results obtained from the calculated heterogeneity, random-effects model and meta-regression were applied in the meta-analysis.

Moreover, the duration of intervention was evaluated by subgroup analysis. A forest plot was correspondingly drawn for all outcomes as the reports of 95\% CI and twosided $P$ value. The narrative form was then employed to pool, if impossible, the findings of articles, including tables and figures. The publication bias was subsequently evaluated by a funnel plot for 10 or more studies enrolled in the meta-analysis. The Begg and Mazumdar rank correlation test was also considered to explore funnel plot asymmetry.

\section{Results}

Study Selection

Following the search, all the identified citations were collated and uploaded into EndNote software and duplicate cases were removed. Full-text studies that had failed to meet the inclusion criteria were also excluded. The results of the search were also presented in a Preferred Reporting Items for Systematic Reviews and Meta-Analyses (PRISMA) flow diagram. The search in the databases led to the identification of a total of 745 potentially relevant articles. Finally, 26 studies (25 RCTs and 1 with a quasiRCT design) examining the effects of physiotherapy and 
PFMEs on the treatment and prevention of pregnancyrelated PFDs, specifically POP, UI, and FI were included in this review $(3,15-39)$ (Figure 1$)$. The sample size varied from 20 to 1800 .

Generation of Results

Physiotherapy and PFMEs for POP Prevention and Treatment

A total of 4 articles selected in this review had explored the effects of physiotherapy and PFMEs on POP prevention and treatment during pregnancy and at postpartum period $(16,17,39)$. In this respect, therapeutic interventions in two studies were PFMEs $(3,17)$. In the study by Bernardes et al (16), both groups had also received interventions, i.e. PFMEs in one group and PFMEs along with hypopressive abdominal exercises (HAEs) in the other group. In the study by Yang et al (39), both groups had received interventions including Kegel exercises and pelvic movements in one group, as well as PFMEs and electrostimulation in the other one. In both articles $(3,17)$, no significant difference was observed between the groups in terms of POP severity or prevalence. In the study by Bernardes et al (16), both groups had also benefitted from an intervention and a significant improvement had been reported in the cross-sectional area of the levator ani muscle and POP compared with that in the controls.

Moreover, Glazener et al (21) in a study with a 12-year follow-up (40) had examined the effects of physiotherapy and PFMEs on postpartum POP treatment. The therapeutic intervention adopted in this study included PFMEs together with bladder exercises. Additionally, no significant difference was observed between the study groups. The POP prevalence in the intervention group had also significantly declined in the study by Yang et al (39).

\section{Physiotherapy and PFMEs for UI Prevention}

Of the studies included in the present review, 9 articles $(23,26,28,29,32-35)$ had investigated the effects of physiotherapy and PFMEs on UI prevention during the 2nd and 3rd trimesters of pregnancy and the postpartum period. As well, 8 studies $(23,26,28,29,32-35)$ had used PFMEs with different protocols for intervention groups. In this respect; Stafne et al (36) had considered a therapeutic exercise program containing aerobic and balance exercises as well as PFMEs for an intervention group.

Out of 9 studies, UI severity or prevalence in the intervention group had significantly improved in 6 cases $(26,29,32-34)$. In three other articles $(28,35)$, no significant difference had been observed between groups in terms of UI prevalence.

\section{Physiotherapy and PFMEs for UI Treatment}

A total number of 9 studies incorporated into this review (15,19-21,24,30,37-39) had shed light on the effects of physiotherapy and PFMEs on UI treatment within the 2nd and 3rd trimesters of pregnancy and during the postpartum period. The therapeutic interventions implemented in

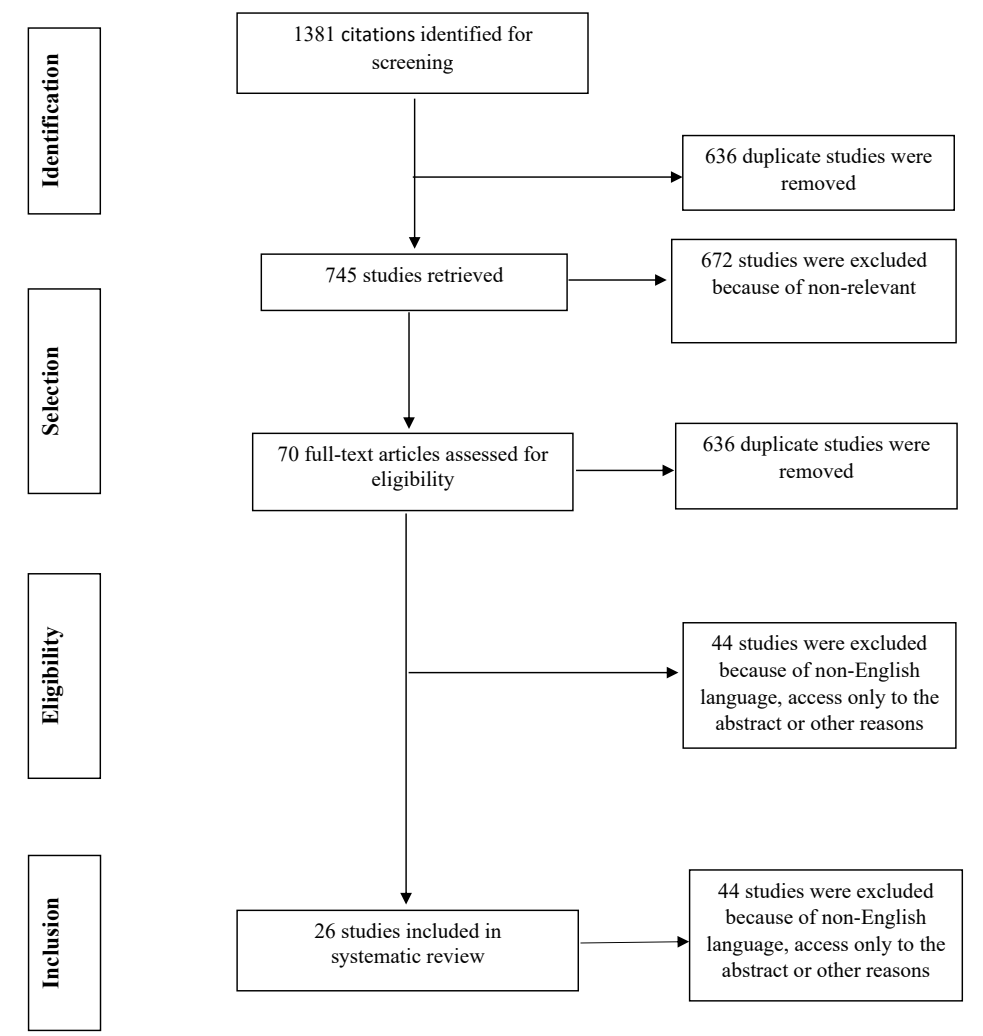

Figure 1. Flow Diagram of Systematic Review for Included. 
three studies were PFMEs $(15,19,38)$, and that was PFMEs and bladder retraining in the study by Glazener et al (21), PFMEs and use of vaginal cones in one study (37), a series of trunk stabilization exercises and PFMEs in the study by Kim et al (24), and transverse abdominal muscle training together with PFMEs in the study by Dumoulin et al (20). In the study by Yang et al (39), both groups had received interventions including Kegel exercises and pelvic movements in the control group as well as PFMEs and electrostimulation in the intervention one. PFMEs along with biofeedback and behavioral therapy had been also considered as the therapeutic interventions used in the study by Oakley et al (3o)

The UI severity or prevalence in the intervention group had significantly improved in four articles $(21,24,37$, 39). In the study by Glazener et al (21), with two followup intervals of $6(41)$ and 12 (40) years, a moderate improvement had been observed in the short-term in UI control in the intervention group (i.e. at a one-year postpartum period); however, it had not persisted after 6 and 12 years. During this period, the UI prevalence had also increased in both groups, which could be attributable to lower levels of motivation to do exercises over time. In three studies $(15,30,38)$, the UI severity or prevalence had been also decreased in both groups, and no significant difference had been reported between both groups. In the study by Dinc et al (19), incontinence severity and prevalence had significantly diminished at pre- and postnatal periods in the intervention group and had subsequently diminished in the postpartum period in the controls. In the article by Dumoulin et al (20) using a 7-year follow-up, no difference was observed in UI severity between intervention and control groups.

Physiotherapy and PFMEs for UI Prevention and Treatment Among the studies selected for this review, a total of 4 articles had questioned the effects of physiotherapy and PFMEs on UI prevention and treatment within the 2nd and 3rd trimesters of pregnancy and during postpartum period $(3,18,22,25)$, in healthy women and patient ones. Accordingly, PFMEs had been examined in three studies $(3,22,25)$, and PFMEs with transverse abdominal muscle contractions and the knack maneuver had been implemented in the study by Chiarelli et al (18). In two studies $(18,25)$, PFMEs had also demonstrated better effects in terms of recovery of incontinence symptoms; however, no significant difference had been observed in other two studies $(3,22)$.

\section{Physiotherapy and PFMEs for FI Prevention}

Only one of the studies (36) included in the present review had investigated the effects of physiotherapy and PFMEs on FI prevention at prenatal and postpartum periods. In this study, therapeutic training sessions including aerobic and balance exercises along with PFMEs had been considered for the intervention group. Following the exercises, FI prevalence in the intervention group had become lower than that of the control group; however, no significant difference had been reported.

\section{Physiotherapy and PFMEs for FI Treatment}

A total of 5 studies in the present review had examined the effects of physiotherapy and PFMEs on FI treatment during pregnancy and at postpartum period $(21,27,30,31)$. The therapeutic interventions were also comprised of PFMEs and bladder exercises in the study by Glazener et al (21), PFMEs and use of vaginal cones in the study by Wilson et al (37), PFMEs together with biofeedback and behavioral therapy in the study by Oakley et al (30), and biofeedback training in the study by Peirce et al (31). In the article by Mahony et al (29), both study groups had received training interventions, i.e. one group had performed PFMEs along with biofeedback and the other group had undergone PFMEs, biofeedback, and electrostimulation. In the study by Mahony et al (29), both groups had demonstrated a significant improvement in terms of FI severity. In the study by Oakley et al (30), FI severity had decreased and no significant difference had been observed between both intervention and control groups. In the study by Peirce et al (31), there was also no significant difference in FI severity between groups. Moreover, in the article by Glazener et al (21), FI prevalence had significantly diminished in the intervention group, but a moderate improvement had been observed in FI control in the intervention group for a short period (i.e. one year following childbirth) which had not persisted after 6 and 12 years. In the study by Wilson et al (37), no significant difference had been similarly reported in terms of IF prevalence between groups.

\section{Meta-Analysis}

In this study, meta-analysis was performed using the CMA (version 2.2.064) on the variable of PFM strength after the end of the intervention via a perineometer. The effect sizes used in these analyzes were also considered as the mean difference values.

According to the results of studies on homogeneity, Cochran's $\mathrm{Q}$ test $(P<0.001)$ and $\mathrm{I}^{2}$ index equal to 90.02 , being larger than the acceptable amount for homogeneity study (25\%), the selected studies were assumed heterogeneous, so random-effects model was implemented to combine their results. The mean differences of the PFM strength between the intervention and control groups following the intervention as the overall effect size based on a random-effects model was 6.94 with a $95 \%$ CI (1.36 to 12.52$)$. The results are presented in Table 1.

Based on Kendall tau's correlation coefficient test (Begg and Mazumdar rank correlation test: Kendall's tau correlation coefficient $=0.6, P=0.22$ ), the publication bias was not also significant; but, in terms of publication bias (i.e. skewed dissemination) using the funnel plot, asymmetry (in terms of the standard error difference of reported averages) indicated skewedness. 
Table 1. Meta-analysis of the Mean Differences Between the Intervention and Control Groups After the Intervention as the Overall Effect Size With a Random Effect Method

\begin{tabular}{|c|c|c|c|c|c|c|c|}
\hline Study Name & Difference in Mean & Standard Error & Variance & Lower limit & Upper limit & $Z$ Value & $P$ Value \\
\hline Kim et al (24) & 17.670 & 3.681 & 13.550 & 10.455 & 24.885 & 4.800 & 0.000 \\
\hline Kahyaoglu et al (23) & 4.200 & 2.253 & 5.076 & -0.216 & 8.616 & 1.864 & 0.062 \\
\hline Wilson et al (37) & 0.000 & 1.477 & 2.181 & -2.895 & 2.895 & 0.000 & 1.000 \\
\hline Dink et al (19) & 15.800 & 3.106 & 9.646 & 9.713 & 21.887 & 5.087 & 0.000 \\
\hline Reilly et al (34) & 1.000 & 1.192 & 1.420 & -1.336 & 3.336 & 0.839 & 0.401 \\
\hline Our study & 6.944 & 2.846 & 8.101 & 1.365 & 12.523 & 2.440 & 0.015 \\
\hline
\end{tabular}

It should be noted that studies with moderate effect sizes had a relative symmetry. In contrast, there were no studies with effect sizes up or down. In this respect, hollow dots indicate the amount of effect sizes reported in existing studies and bold ones represent the amount of effect sizes that should be present or absent in the case of non-skewed publication.

The results were also calculated by the time of intervention, namely pre- and post-natal periods, showing that the combined effect size value with the randomized method on the pregnancy-related interventions (6.54) was less than those in postpartum studies (8.51). It seemed that the time of intervention could be one of the factors affecting heterogeneity of the results of articles.

Subgroup analysis was also fulfilled based on the time of intervention (during pregnancy or at postpartum period as the subgroups). Given that the variance of studies in the subgroups was not equal, a mixed-effects analysis was used to combine their results. Combining the results of the subgroups of the onset of intervention with mixedeffects analysis to find general results by applying the heterogeneity caused by the time of intervention, led to an estimate of 6.85 for the mean differences with 95\% CI between $3.52 \%$ and $12.39 \%$ (Table 2).

With regard to the results of meta-regression analysis to investigate the heterogeneity of the studies, the duration of the intervention was weekly. The results also suggested that the given variable could have a significant effect on the effect sizes reported in the studies $(P=0.002)$. One of the reasons for the heterogeneity of the given articles could be the duration of the intervention, so that the observed effect sizes (namely, the difference between both groups) could decrease as the duration of the intervention had prolonged (Figure 2).

The plot of the meta-regression assessing the effect of the duration of the intervention on the mean differences of PFM strength between the intervention and control groups following the intervention is illustrated in Figure 3.

As well, the results of meta-regression investigating the effect of the duration of the intervention on the mean differences of the PFM strength between the intervention and control groups after the intervention is shown in Table 3.

\section{Discussion}

This systematic review was completed to evaluate RCTs, as well as articles with quasi-RCT designs questioning the effects of physiotherapy and PFMEs on the treatment and prevention of pregnancy-related PFDs specifically POP, UI, and FI.

Since numerous studies had been carried out on this subject, there was a wide variety of sample sizes, different inclusion and exclusion criteria, as well as various methods for treatment protocols and outcome measures. Accordingly, the analysis was very complicated in the present study and the meta-analysis was conducted on only a small number of articles.

There were also many studies conducted on UI adopting various physiotherapy methods, mostly demonstrating significant effects on the improvement of UI symptoms. A systematic review had been further conducted in 2003, which had only included articles using PFMEs for the prevention of pregnancy-related PFDs (5). Besides, Boyle et al in 2012 had fulfilled a Cochrane systematic review and reported that PFMEs could be reflected as effective prevention and treatment strategies for UI and FI in prenatal and postpartum women (7). Another systematic review in 2014 had also evaluated the effects of PFMEs on the prevention and treatment of pregnancy-related UI; suggesting that PFMEs, especially supervised ones

Table 2. Subgroup Analysis Based on Time of Intervention as the Subgroups

\begin{tabular}{lcccccc}
\hline \multirow{2}{*}{ Groups } & \multicolumn{5}{c}{ Effect size and \%95 Confidence Interval } \\
\cline { 2 - 6 } & Number of Studies & Point Estimate & Standard Error & Variance & Lower Limit & Upper Limit \\
\hline Postpartum & 2 & 8.513 & 8.829 & 77.954 & -8.792 \\
Pregnancy & 3 & 6.544 & 3.839 & 14.739 & -0.981 & 14.069 \\
Over all & 5 & 6.857 & 3.521 & 12.396 & -0.043 \\
\hline
\end{tabular}




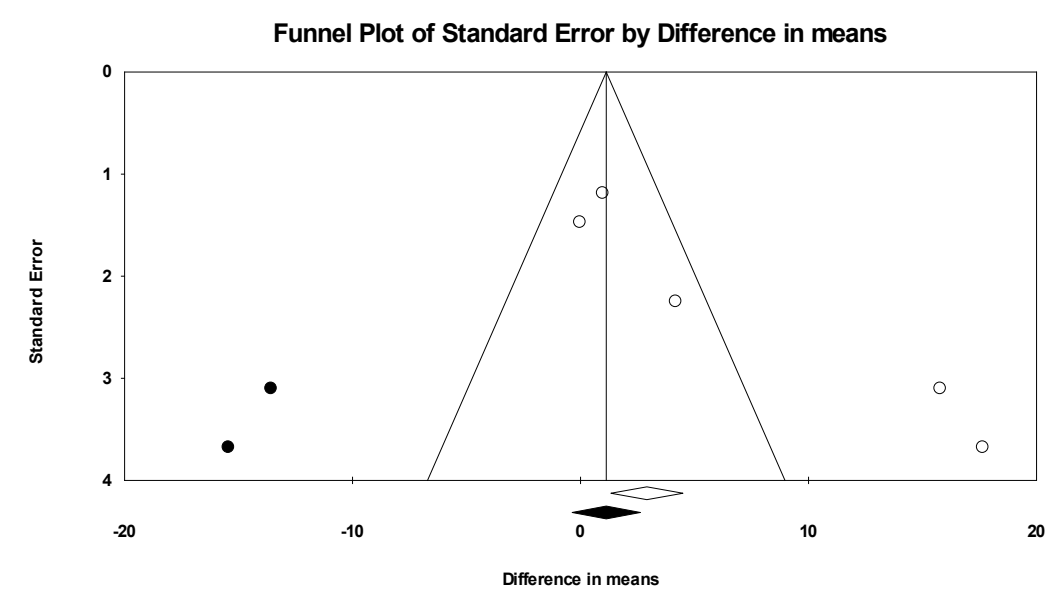

Figure 2. Funnel Plot to Assess Publication Bias.

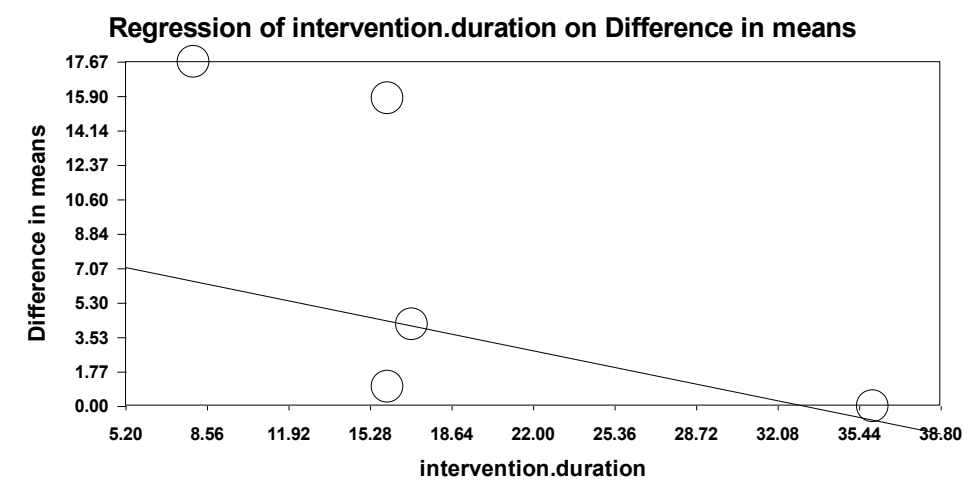

Figure 3. Plot of Meta-Regression to Assess Effect of Intervention Duration on the Mean Differences of the Pelvic Floor Muscle Strength Between the Intervention and Control Groups after the Intervention.

had effectiveness in the prevention and treatment of UI symptoms in postpartum women (42).

According to this systematic review and despite the wide range of methodological differences, most of the selected articles were about patients suffering from UI and there were a small number of studies investigating patients affected with FI and POP. However, most of the articles had concluded that PFMEs and physiotherapy could have much more effects on pregnancy-related UI at least for a short period of time than no interventions and also PFMEs would have better results if they had been intensive and supervised.

Although it was not easy to reach a conclusion regarding the studies examining the effects of physiotherapy and PFMEs on patients with FI and POP, it was suggested to do the same systematic review in the future through adding in more studies about postpartum FI and POP.

Generally, it can be acknowledged that physiotherapy and PFMEs are low-cost and non-invasive interventions with no adverse effects which have been preferred by clinicians over any other invasive procedures.

\section{Conclusions}

According to this systematic review, physiotherapy and PFMEs seem to have effects on pregnancy-related UI although they may not consistently reduce FI or POP severity or prevalence.

\section{Conflict of Interests}

Authors declare that they have no conflict of interests.

Table 3. Results of Meta Regression to Assess the Effect of Intervention Duration on the Mean Differences of the Pelvic Floor Muscle Strength between the Intervention and Control Groups after the Intervention

\begin{tabular}{lccccc}
\hline & Point Estimate & Standard Error & Lower Limit & Upper Limit & Z Value \\
\hline Slope & -0.25432 & 0.08506 & -0.42104 & -0.08760 & -2.98975 \\
Intercept & 8.43159 & 2.01517 & 4.48192 & 12.38126 & 4.18405 \\
Tau-squared & 47.04237 & & & & 0.00279 \\
\hline
\end{tabular}




\section{Ethical Issues}

Not applicable.

\section{Financial Support}

Tabriz University of Medical Sciences supported the study.

\section{Supplementary Materials}

Supplementary file 1. Search Strategy

Supplementary file 2. Extraction Table.

Supplementary file 3. PEDro Scale.

\section{References}

1. Kurdoglu M. Are only anatomic outcomes important after pelvic organ prolapse surgeries? Int J Womens Health Reprod Sci. 2018;6(1):1. doi:10.15296/ijwhr.2018.01

2. Van Geelen H, Ostergard D, Sand P. A review of the impact of pregnancy and childbirth on pelvic floor function as assessed by objective measurement techniques. Int Urogynecol J. 2018;29(3):327-338. doi:10.1007/s00192017-3540-Z

3. Fritel X, de Tayrac R, Bader G, et al. Preventing urinary incontinence with supervised prenatal pelvic floor exercises: a randomized controlled trial. Obstet Gynecol. 2015;126(2):370-377. doi:10.1097/aog.0000000000000972

4. Parés D, Martinez-Franco E, Lorente N, Viguer J, LopezNegre JL, Mendez JR. Prevalence of fecal incontinence in women during pregnancy: a large cross-sectional study. Dis Colon Rectum. 2015;58(11):1098-1103. doi:10.1097/ dcr.0000000000000471

5. Harvey MA. Pelvic floor exercises during and after pregnancy: a systematic review of their role in preventing pelvic floor dysfunction. J Obstet Gynaecol Can. 2003;25(6):487-498. doi:10.1016/s1701-2163(16)30310-3

6. Marzi VL, Serati M. Management of Pelvic Organ Prolapse: Current Controversies. Cham: Springer; 2018.

7. Boyle R, Hay-Smith EJ, Cody JD, Mørkved S. Pelvic floor muscle training for prevention and treatment of urinary and faecal incontinence in antenatal and postnatal women. Cochrane Database Syst Rev. 2012;10:Cd007471. doi:10.1002/14651858.CD007471.pub2

8. $\mathrm{B} \emptyset \mathrm{K}$. Pelvic floor muscle training in treatment of female stress urinary incontinence, pelvic organ prolapse and sexual dysfunction. World J Urol. 2012;30(4):437-443. doi:10.1007/s00345-011-0779-8

9. Ghahremani L, Alipoor M, Amoee S, Keshavarzi S. Health promoting behaviors and self-efficacy of physical activity during pregnancy: an interventional study. Int J Women's Health Reprod Sci. 2017;5(3):181-186. doi:10.15296/ ijwhr.2017.33

10. Hay-Smith J, Berghmans B, Burgio K, et al. Adult conservative management. Incontinence. 2009;4:1025-120.

11. Hung HC, Hsiao SM, Chih SY, Lin HH, Tsauo JY. An alternative intervention for urinary incontinence: retraining diaphragmatic, deep abdominal and pelvic floor muscle coordinated function. Man Ther. 2010;15(3):273279. doi:10.1016/j.math.2010.01.008

12. Mirmolaei ST, Ansari NN, Mahmoudi M, Ranjbar F. Efficacy of a physical training program on pregnancy related lumbopelvic pain. Int J Women's Health Reprod Sci.
2018;6(2):161-166. doi:10.15296/ijwhr.2018.27

13. Sapsford R. Rehabilitation of pelvic floor muscles utilizing trunk stabilization. Man Ther. 2004;9(1):3-12. doi:10.1016/ s1356-689x(03)00131-0

14. Maher CG, Sherrington C, Herbert RD, Moseley AM, Elkins M. Reliability of the PEDro scale for rating quality of randomized controlled trials. Phys Ther. 2003;83(8):713721.

15. Åhlund S, Nordgren B, Wilander EL, Wiklund I, Fridén C. Is home-based pelvic floor muscle training effective in treatment of urinary incontinence after birth in primiparous women? a randomized controlled trial. Acta Obstet Gynecol Scand. 2013;92(8):909-915. doi:10.1111/ aogs. 12173

16. Bernardes BT, Resende AP, Stüpp L, et al. Efficacy of pelvic floor muscle training and hypopressive exercises for treating pelvic organ prolapse in women: randomized controlled trial. Sao Paulo Med J. 2012;130(1):5-9. doi:10.1590/s151631802012000100002

17. Bø K, Hilde G, Stær-Jensen J, Siafarikas F, Tennfjord MK, Engh ME. Postpartum pelvic floor muscle training and pelvic organ prolapse--a randomized trial of primiparous women. Am J Obstet Gynecol. 2015;212(1):38.e31-37. doi:10.1016/j.ajog.2014.06.049

18. Chiarelli P, Cockburn J. Promoting urinary continence in women after delivery: randomised controlled trial. BMJ. 2002;324(7348):1241. doi:10.1136/bmj.324.7348.1241

19. Dinc A, Kizilkaya Beji N, Yalcin O. Effect of pelvic floor muscle exercises in the treatment of urinary incontinence during pregnancy and the postpartum period. Int Urogynecol J Pelvic Floor Dysfunct. 2009;20(10):12231231. doi:10.1007/s00192-009-0929-3

20. Dumoulin C, Martin C, Elliott V, et al. Randomized controlled trial of physiotherapy for postpartum stress incontinence: 7-year follow-up. Neurourol Urodyn. 2013;32(5):449-454. doi:10.1002/nau.22330

21. Glazener CM, Herbison GP, Wilson PD, et al. Conservative management of persistent postnatal urinary and faecal incontinence: randomised controlled trial. BMJ. 2001;323(7313):593-596. doi:10.1136/bmj.323.7313.593

22. Hilde G, Stær-Jensen J, Siafarikas F, Ellström Engh M, $\mathrm{B} ø$ K. Postpartum pelvic floor muscle training and urinary incontinence: a randomized controlled trial. Obstet Gynecol. 2013;122(6):1231-1238. doi:10.1097/ aog.0000000000000012

23. Kahyaoglu Sut H, Balkanli Kaplan P. Effect of pelvic floor muscle exercise on pelvic floor muscle activity and voiding functions during pregnancy and the postpartum period. Neurourol Urodyn. 2016;35(3):417-422. doi:10.1002/ nau. 22728

24. Kim EY, Kim SY, Oh DW. Pelvic floor muscle exercises utilizing trunk stabilization for treating postpartum urinary incontinence: randomized controlled pilot trial of supervised versus unsupervised training. Clin Rehabil. 2012;26(2):132-141. doi:10.1177/0269215511411498

25. Ko PC, Liang CC, Chang SD, Lee JT, Chao AS, Cheng PJ. A randomized controlled trial of antenatal pelvic floor exercises to prevent and treat urinary incontinence. Int Urogynecol J. 2011;22(1):17-22. doi:10.1007/s00192-0101248-4 
26. Kocaöz S, Eroğlu K, Sivashığlu AA. Role of pelvic floor muscle exercises in the prevention of stress urinary incontinence during pregnancy and the postpartum period. Gynecol Obstet Invest. 2013;75(1):34-40. doi: $10.1159 / 000343038$

27. Mahony RT, Malone PA, Nalty J, Behan M, O'Connell P R, O'Herlihy C. Randomized clinical trial of intraanal electromyographic biofeedback physiotherapy with intra-anal electromyographic biofeedback augmented with electrical stimulation of the anal sphincter in the early treatment of postpartum fecal incontinence. Am J Obstet Gynecol. 2004;191(3):885-890. doi:10.1016/j. ajog.2004.07.006

28. Mason L, Roe B, Wong H, Davies J, Bamber J. The role of antenatal pelvic floor muscle exercises in prevention of postpartum stress incontinence: a randomised controlled trial. J Clin Nurs. 2010;19(19-20):2777-2786. doi:10.1111/ j.1365-2702.2010.03297.x

29. Mørkved S, Bø K, Schei B, Salvesen KA. Pelvic floor muscle training during pregnancy to prevent urinary incontinence: a single-blind randomized controlled trial. Obstet Gynecol. 2003;101(2):313-319. doi:10.1016/s0029-7844(02)02711-4

30. Oakley SH, Ghodsi VC, Crisp CC, et al. Impact of pelvic floor physical therapy on quality of life and function after obstetric anal sphincter injury: a randomized controlled trial. Female Pelvic Med Reconstr Surg. 2016;22(4):205213. doi:10.1097/spv.0000000000000255

31. Peirce C, Murphy C, Fitzpatrick M, et al. Randomised controlled trial comparing early home biofeedback physiotherapy with pelvic floor exercises for the treatment of third-degreetears(EBAPT Trial). BJOG. 2013;120(10):12401247; discussion 1246. doi:10.1111/1471-0528.12194

32. Pelaez M, Gonzalez-Cerron S, Montejo R, Barakat R. Pelvic floor muscle training included in a pregnancy exercise program is effective in primary prevention of urinary incontinence: a randomized controlled trial. Neurourol Urodyn. 2014;33(1):67-71. doi:10.1002/nau.22381

33. Reilly ET, Freeman RM, Waterfield MR, Waterfield AE, Steggles P, Pedlar F. Prevention of postpartum stress incontinence in primigravidae with increased bladder neck mobility: a randomised controlled trial of antenatal pelvic floor exercises. BJOG. 2002;109(1):68-76. doi:10.1111/j.1471-0528.2002.t01-1-01116.x

34. Sangsawang B, Sangsawang N. Is a 6-week supervised pelvic floor muscle exercise program effective in preventing stress urinary incontinence in late pregnancy in primigravid women?: a randomized controlled trial. Eur J Obstet Gynecol Reprod Biol. 2016;197:103-110. doi:10.1016/j. ejogrb.2015.11.039

35. Sleep J, Grant A. Pelvic floor exercises in postnatal care. Midwifery. 1987;3(4):158-164. doi:10.1016/s02666138(87)80035-9

36. Stafne SN, Salvesen KÅ, Romundstad PR, Torjusen IH, Mørkved S. Does regular exercise including pelvic floor muscle training prevent urinary and anal incontinence during pregnancy? a randomised controlled trial. BJOG. 2012;119(10):1270-1280. doi:10.1111/j.14710528.2012.03426.x

37. Wilson PD, Herbison GP. A randomized controlled trial of pelvic floor muscle exercises to treat postnatal urinary incontinence. Int Urogynecol J Pelvic Floor Dysfunct. 1998;9(5):257-264. doi:10.1007/bf01901501

38. Woldringh $\mathrm{C}$, van den Wijngaart $\mathrm{M}$, Albers-Heitner $\mathrm{P}$, Lycklama à Nijeholt AA, Lagro-Janssen T. Pelvic floor muscle training is not effective in women with UI in pregnancy: a randomised controlled trial. Int Urogynecol J Pelvic Floor Dysfunct. 2007;18(4):383-390. doi:10.1007/ s00192-006-0175-x

39. Yang S, Sang W, Feng J, et al. The effect of rehabilitation exercises combined with direct vagina low voltage low frequency electric stimulation on pelvic nerve electrophysiology and tissue function in primiparous women: a randomised controlled trial. J Clin Nurs. 2017;26(23-24):4537-4547. doi:10.1111/jocn.13790

40. Glazener CM, MacArthur C, Hagen S, et al. Twelveyear follow-up of conservative management of postnatal urinary and faecal incontinence and prolapse outcomes: randomised controlled trial. BJOG. 2014;121(1):112-120. doi:10.1111/1471-0528.12473

41. Glazener CM, Herbison GP, MacArthur C, Grant A, Wilson PD. Randomised controlled trial of conservative management of postnatal urinary and faecal incontinence: six year follow up. BMJ. 2005;330(7487):337. doi:10.1136/ bmj.38320.613461.82

42. Mørkved S, Bø K. Effect of pelvic floor muscle training during pregnancy and after childbirth on prevention and treatment of urinary incontinence: a systematic review. Br J Sports Med. 2014;48(4):299-310. doi:10.1136/ bjsports-2012-091758

(c) 2020 The Author(s); This is an open-access article distributed under the terms of the Creative Commons Attribution License (http:// creativecommons.org/licenses/by/4.0), which permits unrestricted use, distribution, and reproduction in any medium, provided the original work is properly cited. 